\title{
O LUGAR DA BIOQUÍMICA NO PROCESSO DE CUIDAR: VISÃO DE GRADUANDOS EM ENFERMAGEM
} Rodrigo Grazinoli Garrido” Fernanda Oliveira Araújo², Thiago Henrique Oliveira²; Fabiola de
Sampaio Rodrigues Grazinoli Garrido ${ }^{3}$

1 Perito Criminal, IPPGF/PCERJ; 2 Estudante de Enfermagem UFBA- IMS/CAT, bolsista do Programa Permanecer; 3 Professora Bioquímica UFBA - IMS/CAT

\section{Resumo:}

A bioquímica é uma disciplina fundamental para cursos da área de saúde. Esses alunos devem estar aptos a atuar em um meio onde o domínio das reações orgânicas é imprescindível para compreender a dialética saúde-doença. Isto é especialmente importante na Enfermagem, onde se exige capacidade de investigação, diagnóstico, planejamento, implementação e avaliação do processo de cuidar. Com isso, a bioquímica deve buscar vincular o conteúdo à atividade profissional, com ênfase fisiopatológica, sem descuidar dos seus fundamentos. Se, por um lado, a primeira opção torna o ensino da disciplina mais atraente e interessante, uma sólida formação básica habilitaria o estudante a analisar sua prática sob um aspecto mais técnico, adaptando-se a novas circunstâncias e tecnologias. Nesse contexto, buscou-se descrever a opinião de estudantes de Enfermagem da UFBA acerca da importância da disciplina para a futura profissão e verificar a percepção quanto ao próprio rendimento na disciplina e a capacidade de relacionar conceitos da bioquímica à fisiopatologia. O interesse dos alunos se dividiu entre o entendimento do metabolismo e seus distúrbios, concorrendo para o diagnóstico de patologias. De modo geral, os alunos mostraram bom desempenho na disciplina. Assim, tornou-se impossível pensar a bioquímica desvinculada da fisiopatologia. Este viés é buscado pelos próprios alunos, favorecendo desempenho no curso.

Palavras-chave: ensino, graduação, distúrbios metabólicos,

\begin{abstract}
:
Biochemistry is a basic subject for health courses. These students should be able to work in an environment where organic reactions knowledge is essential to understand the healthdisease dialectic. It is especially important in nursing, which requires the ability to investigate, diagnose, planning, implementation and evaluation of care process. Because of this, biochemistry should seek to link content to professional activity, with emphasis on pathophysiology, without ignoring its own objectives. Once the first option makes teaching a more attractive and interesting subject, on the other hand, a solid basic training would enable students to consider its work under a more technical view, adapting to new circumstances and technologies. In this context, we attempted to describe the UFBA's nursing students' views about the subject importance for the future profession and to verify the perception of their own advances in the discipline and ability to relate concepts of biochemistry and pathophysiology. Students' interest was divided between metabolism understanding and its disorders, contributing for the diseases diagnosis. Overall, students showed good subject performance. Thus, it became impossible to think biochemistry devoid of pathophysiology. This view is claimed by the students themselves, encouraging performance in the course.
\end{abstract}

Keywords: teaching, undergraduate, metabolic disorders 


\section{INTRODUÇÃO}

A bioquímica é um exemplo de disciplina do ciclo básico oferecida em praticamente todos os cursos da área de saúde $[1,2,3]$. Ela atende a grupos muito heterogêneos de alunos [4] e sua característica multidisciplinar é um indicativo da sua imperiosa aplicação nos mais diversos campos de atuação profissional [1].

O aumento do conhecimento nas diversas áreas das Ciências Biológicas, na bioquímica e na biologia molecular, em particular, tem causado dilema para os professores envolvidos com essas áreas: enquanto o conhecimento aumenta, é impossível aumentar proporcionalmente a carga horária das disciplinas [5]. Nem mesmo os livros-texto mais atuais são capazes de acompanhar a quantidade de informação produzida anualmente [6].

De modo geral, os profissionais da saúde atuam em um cenário em que o domínio das potenciais reações orgânicas é imprescindível para o procedimento frente às mais variadas situações e patologias. O entendimento dos distúrbios metabólicos ou a interpretação de exames clínicos demandam o uso do conhecimento discutido em disciplinas como a bioquímica [1]. Isto se torna especialmente importante na prática da enfermagem, onde, atualmente, além de se exigir a prática assistencial, para desenvolver a arte de cuidar e de ensinar a cuidar, tornaram-se essenciais a pesquisa e o diagnóstico [7].

Apesar disso, os estudantes de enfermagem mostram, em geral, bastante dificuldade e desinteresse pelas disciplinas de biociências $[8,9,10]$. A fim de superar este problema, muitas propostas têm sido testadas no ensino de bioquímica $[8,9]$. Vincular o conhecimento de bioquímica às outras áreas do curso, especialmente ao ciclo profissionalizante e à prática do cuidar, tem mostrado sucesso $[10,11]$ Certamente, a ampliação das discussões contextualizadas fornece aos alunos bons resultados porque nesse ambiente de ensino-aprendizagem, a motivação dos educandos é maior [12].

Nesse contexto, a partir de questionários objetivos aplicados a alunos do curso de Enfermagem de II, IV, VI e VII semestres da Universidade Federal da Bahia (UFBA) campus Anísio Teixeira, destacou a opinião dos estudantes acerca da importância da disciplina bioquímica para a futura profissão. Além de verificar a percepção dos alunos quanto ao próprio rendimento na disciplina e a capacidade de relacionar conceitos de biomoléculas, metabolismo e desordens metabólicas.

\section{MÉTODO}

No ano de 2007, foi elaborado e aplicado aos alunos que terminavam o I e III semestres do curso de Enfermagem do Campus Anísio Teixeira do Instituto Multidisciplinar em Saúde da Universidade Federal da Bahia (IMS/CAT/UFBA), na cidade de Vitória da Conquista um questionário transversal com quatro perguntas objetivas (importância da disciplina para a formação profissional, rendimento na disciplina, conhecimento sobre distúrbios metabólicos, bem como o envolvimento da bioquímica no processo de cuidar). Os questionários não eram identificados. Com exceção da questão sobre o rendimento na disciplina, com respostas mutuamente excludentes, as outras questões permitiam mais do que uma resposta.

$\mathrm{Na}$ época, apenas duas turmas, com um total de 80 alunos, cursavam Enfermagem no recém-criado IMS/CAT/UFBA. Responderam ao questionário 61 (37 do I semestre e 24 do III semestres) estudantes, o que comporta um erro amostral de cerca de $6 \%$. A disciplina bioquímica é ministrada durante o primeiro período do curso. Os resultados foram analisados apenas de forma descritiva e apresentados em percentuais. 


\section{RESULTADOS E DISCUSSÃO}

A importância da disciplina foi atribuída à formação de base para a atuação profissional (Tabela 1). O fato de estar colocada como pré-requisito em um currículo estruturado não representou a importância da disciplina para os estudantes.

Muitas iniciativas têm sido desenvolvidas para tornar o ensino de bioquímica mais atraente e interessante para diversos públicos e, em especial, para enfermagem [8,9], entre elas, a vinculação dos conteúdos de bioquímica a todas às outras disciplinas de fases mais avançadas da formação $[10,11]$. Tais iniciativas vinculam o conteúdo ao cotidiano e ao interesse dos profissionais. Assim, pode-se concluir que a contribuição dos conhecimentos de bioquímica tornou-se maior do que o fato desta ser pré-requisito para outras disciplinas (Tabela 1). Esta visão é compartilhada por estudantes de Fisioterapia [3].

Tabela 1 - Importância da disciplina para a formação do aluno, segundo a opinião dos estudantes dos semestres I e III de enfermagem da UFBA.

\begin{tabular}{c|cc}
\multicolumn{1}{c|}{ Importância } & \multicolumn{2}{c}{ Percentual } \\
\hline $\begin{array}{c}\text { Como pré-requisito de outras } \\
\text { sem I }\end{array}$ & 5 \\
$\begin{array}{c}\text { disciplinas } \\
\text { Para facilitar entendimento de reações }\end{array}$ & 3 & 36 \\
$\begin{array}{c}\text { metabólicas } \\
\text { Para o entendimento de distúrbios }\end{array}$ & 31 & 25 \\
$\begin{array}{c}\text { metabólicos } \\
\text { Para diagnóstico de patologias }\end{array}$ & 32 & 33 \\
\hline \multicolumn{1}{r}{ Total } & $\mathbf{1 0 0}$ & $\mathbf{1 0 0}$ \\
\hline
\end{tabular}

$\mathrm{Na}$ Tabela 1, pode-se considerar que a visão dos alunos da UFBA apresentou-se dividida entre o foco no entendimento do metabolismo como um todo e dos distúrbios metabólicos, concorrendo para o diagnóstico de patologias. Em primeiro instante, Pellizon et al. [13] defendem que o processo de enfermagem deve focalizar o indivíduo como um todo, e não apenas a doença. Dessa forma, a abordagem na disciplina na UFBA pareceu bastante adequada à visão atual de atuação do enfermeiro.

De modo geral, os alunos apresentaram como essencial a aplicação dos conhecimentos bioquímicos para o diagnóstico de patologias. A tabela 2 apresenta diabetes mellitus e fenilcetonúria como patologias mais citadas por alunos de distintos períodos, quando se questionava o conhecimento acerca de distúrbios metabólicos. O interesse dos alunos pela Bioquímica das Doenças é notório também no México [14], sendo o diabetes mellitus um dos tópicos mais representativos. Talvez este interesse se deva à posição do Diabetes como uma das desordens endócrinas mais comuns atualmente. Isso pode explicar o destaque por parte dos alunos. Reconhecidamente, o diabetes tem se tornado um grande desafio à saúde no mundo. De acordo com projeções, ela alcançará 220 milhões de pessoas até 2010 e ultrapassará 300 milhões até 2025 [15]. 
Tabela 2 - Patologia relacionada a distúrbio metabólico pelos alunos dos semestres I e III de enfermagem da UFBA.

\begin{tabular}{c|cc}
\hline Patologia & \multicolumn{2}{|c}{ Percentual } \\
& sem I & sem III \\
\hline Fibrose cística & 17 & 21 \\
Diabetes Mellitus & 39 & 30 \\
Fenilcetonúria & 44 & 49 \\
\hline Total & $\mathbf{1 0 0}$ & $\mathbf{1 0 0}$ \\
\hline
\end{tabular}

A distribuição de rendimento na componente curricular (Tabela 3 ) foi equivalente em ambos os períodos da UFBA, estando fortemente fixada no nível bom. O bom rendimento apontado pela maioria dos alunos foi bastante surpreendente, uma vez que de modo geral, os estudantes de enfermagem mostram certa dificuldade em disciplinas de biociências, principalmente quando estas demandam conhecimentos básicos de química, física e biologia $[3,9,10]$.

Tabela 3 - Rendimento obtido na disciplina por estudantes dos semestres I e III de Enfermagem da UFBA.

\begin{tabular}{|c|c|c|}
\hline \multirow{2}{*}{ Rendimento } & \multicolumn{2}{|c|}{ Percentual } \\
\hline & sem I & sem III \\
\hline péssimo & 8 & 4 \\
\hline ruim & 22 & 21 \\
\hline bom & 62 & 58 \\
\hline ótimo & 8 & 17 \\
\hline Tot & 100 & 100 \\
\hline
\end{tabular}

Nesse contexto parece estar presente o direcionamento dos trabalhos pelo professor, o interesse intrínseco dos alunos e a proximidade dos conteúdos com a prática profissional, como já observado por outros autores [2]. Pois, segundo Campbell e Leathard [10], estudantes de enfermagem do Reino Unido mostravam conhecimento muito baixo sobre princípios das ciências básicas e de biologia. Todavia, quando se tratam de questões relacionadas à pratica (fisiopatologia), os resultados foram melhores. Mesmo assim, de acordo com os autores, estes resultados poderiam trazer implicações para a qualidade no processo de cuidar e no desenvolvimento da profissão. Pois, esses profissionais apresentariam habilidade limitada de resolver situações clínica, de se adaptar a novas circunstâncias e de entender a prática.

É certo que, mediante a importância atribuída à disciplina (Tabela 1), bem como o já esperado interesse pela aplicação de conhecimentos bioquímicos à Diabetes mellitus [14], foi proposto aos alunos analisar um caso particular sobre esta patologia. Nesse nível de conhecimento, foi possível fazer inferências sobre hábitos e atitudes encerrados no foco desses estudantes: o processo de cuidar (Tabela 4).

A maioria (62\%), a partir dos conhecimentos adquiridos na disciplina de Bioquímica, recomendaria uma dieta aos pacientes diabéticos. Um menor número recomendaria exercícios físicos ou, ainda, a não ingestão de bebidas alcoólicas.

De fato, uma das características básicas do progresso dos estudantes deve-se à forma empregada pelos alunos para explicarem as situações que os cercam [16]. Assim, Oliveira et al [2] observaram o entusiasmo pela bioquímica entre os alunos de odontologia e atribuíram tal fato à observação pelos alunos da aplicação cada vez maior da disciplina nas novas descobertas da ciência moderna, alterando inclusive o mercado de trabalho. 
Tabela 4 - Recomendação dos estudantes dos semestres I e III de enfermagem da UFBA a pacientes diabéticos, utilizando-se dos conhecimentos bioquímicos no processo de cuidar de.

\begin{tabular}{|c|c|c|}
\hline \multirow{2}{*}{ Aplicação no processo de cuidar } & \multicolumn{2}{|c|}{ Percentual } \\
\hline & sem I & sem III \\
\hline recomendaria não ingerir bebida alcoólica & 15 & 21 \\
\hline recomendaria exercício físico & 23 & 17 \\
\hline desenvolveria uma dieta adequada & 62 & 62 \\
\hline Total & 100 & 100 \\
\hline
\end{tabular}

\section{Conclusão}

Foi possível verificar que os estudantes de enfermagem do IMS/UFBA encontram na disciplina de Bioquímica uma forte relação do conteúdo abordado com a realidade do processo de cuidar. O fato esteve implícito na importância da bioquímica enquanto fornecedora de conteúdo atualizado e próximo da realidade profissional desses estudantes, permitindo que os estudantes, mesmo aqueles que não consideram o rendimento na disciplina aceitável, inferissem sobre a aplicação desse conhecimento no processo de cuidar do Diabetes mellitus. De modo geral, o viés fisiopatológico é buscado próprios alunos de Enfermagem na disciplina de Bioquímica.

\section{Agradecimento}

Ao Programa Permanecer/UFBA pelas bolsas que visam à manutenção e permanência acadêmica dos alunos em dificuldades sócio-econômicas.

\section{REFERÊNCIAS BIBLIOGRÁFICAS}

[1] Gomes, K.V.G. e Rangel, M. (2006) Relevância da Disciplina Bioquímica em Diferentes Cursos de Graduação da UESB, na Cidade de Jequié. Rev. Saúde.Com; 2(1), 161-168.

[2] R.C. de Oliveira; F.G. Iano; T.L. da Silva, M.A.F. Buzalaf (2007) Percepção dos Alunos do Curso de Odontologia de uma Universidade Brasileira em Relação à Importância da Disciplina de Bioquímica na sua Profissão. RBEBBM nº1 Artigo B, 1-6.

[3] T.D.L. Pinheiro; J.A. da Silva; P.R.M. de Souza; M.M. do Nascimento; H.D. Oliveira (2009) Ensino de Bioquímica para acadêmicos de Fisioterapia: visão e avaliação do discente. RBEBBM no1, Artigo C, 1-11.

[4] P.F. Beckhauser; E.M. Almeida; A.L.B. Zeni (2006) O universo discente e o ensino de Bioquímica. RBEBBM n. ${ }^{\circ}$ 02, artigo $B$, 01-07.

[5] W.C.B. Regis; M.L.G.S., Gonçalves; M.M. Santoro (2001) Avaliação das potencialidades de um curso prático de bioquímica na implementação do ensino teórico em cursos profissionalizantes. RBEBBM Especial 1.

[6] L. De Meis (1998) Ciência e Educação. O Conflito Humano-tecnológico. Ed. do Autor. Rio de Janeiro, 198p.

[7] V. Carvalho (2004) Cuidando, pesquisando e ensinando: acerca de significados e implicações da prática da enfermagem. Rev. Latino-americana de Enfermagem, 12(5).

[8] D.K., Yokaichiya; E., Galembeck; B.B., Torres (2004) O que alunos de diferentes cursos procuram em disciplinas extracurriculares de bioquímica? RBEBBM no 1, E2. 
[9] S. Davies; F. Murphy; S. Jordan (2000) Bioscience in the pre-registration curriculum: finding the right teaching strategy. Nurse Education Today, 20, 123-135.

[10] J. Campbell; H. Leathard (2008) Nurses' knowledge of biological and related science. NTresearch, vol. 5, n05, 372-380.

[11] I.F. Silva; N.A. Batista (2003) Biochemistry in Undergraduate Health Courses. Biochemistry and Molecular Biology Education, vol 31, n6, 397-401.

[12] J. Kieser, P. Herbison, T. Harland (2005) The influence of context on students' approaches to learning: a case study. Eur. J. Dent. Educ., 9, 150-156.

[13] F. Pellisnon; M.M. Nagumo; E.S. Cunha; L.deL. Melo (2007) Aplicação prática do processo de enfermagem a uma adolescente portadora de doença crônica. Rev. Esc. Enferm. USP.41(3), 513-517.

[14] R.H. Glew; D.L. Vander Jagt (2001) A Biochemistry of Human Disease course for undergraduate and graduate students. Bioc. and Mol. Biol. Educ., vol. 29, 188-192.

[15] E. Adeghate; P. Schatner; E. Dunn (2006) Un Update on the Etiology and Epidemiology of Diabetes Mellitus. Ann. N.Y. Acad. Sci. 1084, 1-29.

[16] N. Bizzo (2002) Ciências: fácil ou difícil? Série Palavra de Professor, Editora Ática, 2a ed., 5 a reimp., $144 \mathrm{p}$. 